\title{
A case of didelphic uterus and blind hemivagina with renal dysplasia and ectopic ureter presenting with vulvodynia and recurrent fever
}

\author{
Elke Stevens • Jan Baekelandt • Lutgarde Lemmens • \\ Els Dufraimont • André Valcke • Marcel De Bruyn • \\ Jan Bosteels
}

Received: 25 December 2008 /Revised: 31 January 2009 /Accepted: 2 February 2009 / Published online: 14 February 2009

(C) Springer-Verlag 2009

\begin{abstract}
Didelphic uterus with blind hemivagina and ipsilateral renal anomaly is a rare congenital malformation. The pathogenesis of this syndrome and its embryologic origin are still the subject of discussion. Due to the variable clinical picture and low index of suspicion, diagnosis is often delayed or inaccurate. Conservative surgical treatmentexcision of the obstructing vaginal septum and marsupialization of the blind hemivagina-is considered the most appropriate treatment. This report describes the case of a 16-year-old girl with didelphic uterus and double vagina, with obstruction of the left hemivagina. The patient was also diagnosed with left renal dysplasia and ipsilateral ureter communication with the obstructed vagina. Following various examinations and left uretero-nephrectomy, the patient was effectively treated with resection of the vaginal septum.
\end{abstract}

Keywords Didelphic uterus - Blind hemivagina .

Müllerian anomalies · Ectopic ureter $\cdot$ Renal anomaly

\section{Introduction}

Female genital tract development anomalies represent a broad spectrum of pathologies that are frequently misdiag-

The corresponding author certifies that there is no actual or potential conflict of interest in relation to this article.

E. Stevens $\cdot$ J. Baekelandt $\cdot$ L. Lemmens $\cdot$ E. Dufraimont $\cdot$

A. Valcke $\cdot$ M. De Bruyn $\cdot$ J. Bosteels $(\bowtie)$

Department $\mathrm{Ob} / \mathrm{Gyn}$, Imeldahospital,

Imeldalaan 9,

2820 Bonheiden, Belgium

e-mail: jan.bosteels@imelda.be nosed leading to suboptimal treatment. The prevalence may be underestimated because asymptomatic cases can remain undiagnosed and normal reproductive outcome is still possible. Didelphic uterus with blind hemivagina is a rare congenital malformation that is ascribed to a failure in the fusion process of the two Müllerian ducts. It almost invariably coexists with anomalies of the ipsilateral mesonephric derivates (kidney and ureter) because of their close embryological relationship. The reported incidence of uterus didelphys with renal agenesis in the general population is estimated within the range of $0.1 \%$ to $3.8 \%$ [1]. Early and accurate diagnosis can be difficult; however, prompt diagnosis and simple excision of the obstructive vaginal septum can completely relieve the symptoms and prevent further sequelae.

\section{Case report}

A 16-year-old girl presented with lower abdominal discomfort, vulvodynia, purulent vaginal discharge, and a fever of $38.5^{\circ} \mathrm{C}$ at the time of menstruation. Because of a history of dysmenorrhea, menorrhagia, and intermenstrual bleeding, she used Cyclocur ${ }^{\circledR}$ (a sequential preparation of estradiol $2 \mathrm{mg}$ and norgestrel $0.5 \mathrm{mg}$ ) which was started after trying a classic oral contraceptive. Initially, a diagnosis of acute salpingo-oophoritis type II was made and oral antibiotic treatment (amoxicillin-clavulanic acid 500/125 mg, three times daily) started at the emergency room by the gynecologist on call. A follow-up consultation was scheduled. Microbiological culture of the vaginal swab demonstrated the growth of Escherichia coli.

The patient was born with congenital anomalies of both hands and her left leg, which were ascribed to amniotic 
band sequence. These malformations were treated by orthopedic surgery.

After 4 days of treatment, she still suffered from a fever of $38^{\circ} \mathrm{C}$ with a C-reactive protein (CRP) of $13 \mathrm{mg} / \mathrm{dl}$ (normal range $<0.5 \mathrm{mg} / \mathrm{dl}$ ). Examination with a small speculum showed no abnormalities. Because the patient was virgin, a bimanual pelvic examination was not performed. The patient was admitted to continue effective intravenous (IV) antibiotic therapy (amoxicillin-clavulanic acid $1 \mathrm{~g}$, three times daily) and to investigate underlying pathology.

Transabdominal ultrasonography showed an abnormal uterine position, a possibly infected structure of $8.3 \times 5.6 \mathrm{~cm}$ on the posterior left side of the bladder, and a very small left kidney. An MRI was performed for more accurate diagnosis. The radiologist reported a uterus with two cavities and one cervix and a tortuous dilated hydro-ureter on the left side, with possible connection to the cervix. The left kidney consisted of a pyelon with almost absent atrophic cortex. The initial diagnosis was a congenital uterine anomaly, possibly uterus bicornis unicollis complicated by an infected left hydro-ureter with ectopic isthmic ending and severely dysplastic left kidney. Microbiological examinations of the urine and vaginal swabs were at that time negative. The patient was discharged on oral amoxicillin-clavulanic acid with a scheduled follow-up consultation. A diagnostic and possible operative laparoscopy after cooling of the inflammatory process was scheduled.

One week after discontinuation of antibiotic therapy, the patient was re-admitted with high fever (up to $38.8^{\circ} \mathrm{C}$ ). Blood tests revealed an elevated CRP of $18 \mathrm{mg} / \mathrm{dl}$. Antibiotic therapy IV (initially levofloxacin $500 \mathrm{mg}$ daily, switched to piperacillin-tazobactam $4 \mathrm{~g} / 500 \mathrm{mg}$ four times daily because of increasing CRP) was again started, lasting until the scheduled laparoscopic procedure.

Three weeks after the first hospital admission, a laparoscopic uretero-nephrectomy was performed by an experienced gynecological endoscopist assisted by a urologist. Microscopic examination of the resected specimen revealed a ureter with extensive chronic inflammation. The patient recovered well from the procedure and was discharged on day 5 after surgery. However, 4 weeks later she presented with the same complaints as at her initial emergency room consultation. Microbiological examination of a vaginal swab demonstrated significant growth of $E$. coli. Another MRI scan revealed a comparable sacculary dilated infected structure in the left hemipelvis as seen earlier (Figs. 1 and 2). A uterus bicornis unicollis with right horn communicating with the endocervix and left horn communicating solely with the left pyoureter ectopical ending in the cervix was proposed by the consulting radiologist as an initial diagnosis. A small connection between the cervix and sacculary structure on the left, believed to be the dilated ureter, was noted.

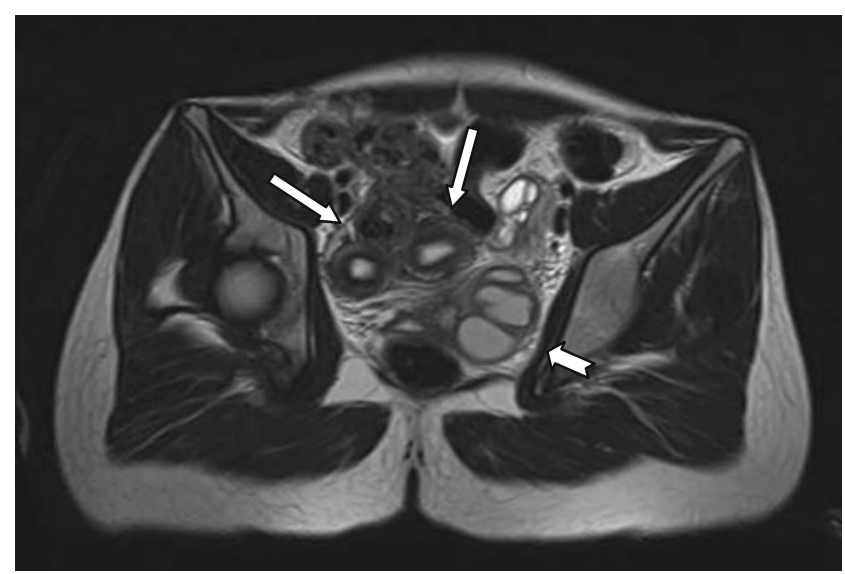

Fig. 1 MRI scan (paracoronal T2-weighted image) showing the tortuous sacculary dilated structure in the left hemipelvis (thick arrow) and the two uterine cavities (thin arrows)

After multidisciplinary discussion of these findings among the primary care physician, radiologist, urologist, and gynecological staff, a hysteroscopic examination under general anesthesia was scheduled. A vaginoscopic hysteroscopy was performed using a single-channel Bettocchi $3.5 \mathrm{~mm} 30^{\circ}$ hysteroscope with the use of normal saline as distension medium with a bag and a cuff pressure of $100 \mathrm{mmHg}$. This hysteroscopy was performed by a senior gynecologist with expertise in outpatient vaginoscopic hysteroscopy. The examination revealed a normal endocervix and a normal uterine corpus on the right. The release of a brownish purulent material was incidentally caused upon manipulation of the left side. A small opening from a slightly bulging blind left vagina was noted and the hysteroscope was inserted through this opening. After rinsing of the entered pouch, a second flattened cervix

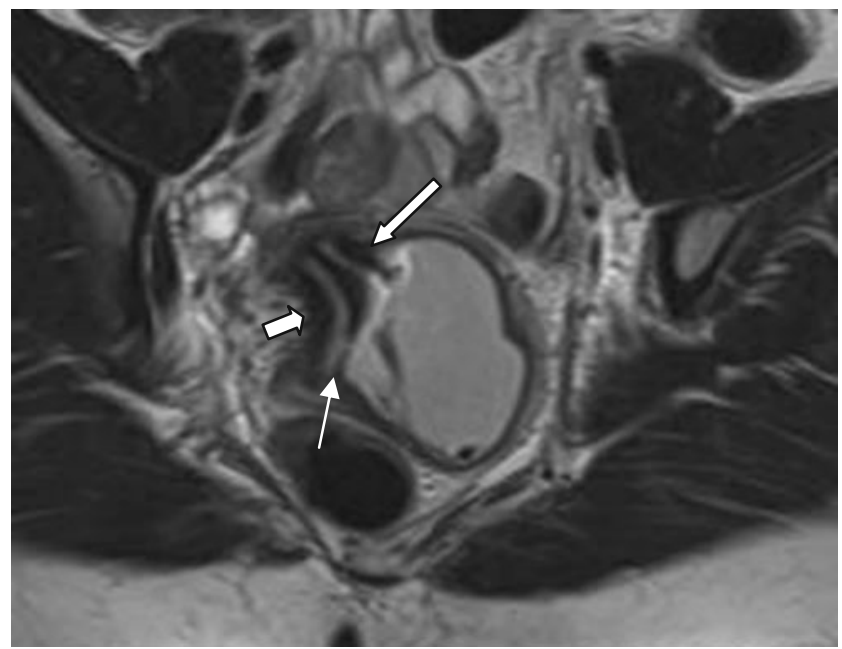

Fig. 2 MRI scan (paracoronal T2-weighted image) before excision of the obstructive septum: left cervix (long arrow) draining into the blind hemivagina and right cervix (short arrow) draining to normal right hemivagina, with connection between the two hemitracts (thin arrow) 
was visualized, giving easy access of the hysteroscope into a normal left hemi uterus.

A final and correct diagnosis of didelphic uterus with obstructed hemivagina and ipsilateral ectopic ureter with dysplastic kidney was made. The vaginal septum was easily excised with marsupialization of the margins. A Foley catheter was left in the former blind left hemivagina for 5 days to establish further patency. A third postoperative MRI scan showed the collapsed sacculary collection with the Foley catheter and the collapsed ureteral remnant, ending in the now open left vagina (Fig. 3).

After the next menstrual cycle, a follow-up MRI was performed confirming the didelphic uterus. It showed the left uterus and ureteral remnant draining into a pouch that ends in the vagina, through a small channel, and through a larger connection, which was the surgically made foramen between the right hemivagina and previously closed left hemivagina.

After 1 year of follow-up, the patient is still asymptomatic with a normal menstrual cycle without hormonal therapy.

\section{Discussion}

Didelphic uterus with blind hemivagina is a rare congenital malformation that usually coexists with malformations of the urinary tract such as renal agenesis, hypoplasia, dysplasia, or ectopic ureter [2, 3]. Uterus didelphys represents $11.1 \%$ of uterine anomalies and in most cases a vaginal septum is present. Urinary tract anomalies are present in $23 \%$ of these women [4]. The reported incidence of uterus didelphys with renal agenesis in the general population is low: $0.1-3.8 \%$ [1]. This may be an underestimate due to inaccurate diagnoses and because some

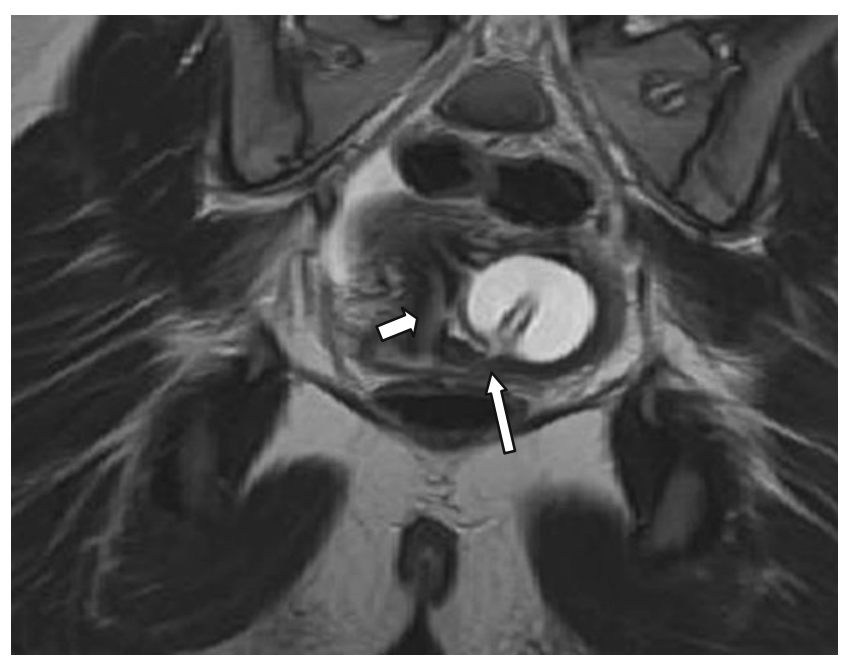

Fig. 3 MRI scan (paracoronal T2-weighted image) after excision of the septum: Foley catheter in the collapsed pouch, draining into the now open left vagina (long arrow). Right cervix draining to the vagina (short arrow) with connection to the vaginal pouch on the left defects remain undetected. This also explains the discrepancies in reported incidences.

Most uterine anomalies result from a defect in the development or fusion of the paired Müllerian ducts during embryogenesis. Complete failure of fusion of the paired Müllerian ducts results in duplication of the uterine corpus and cervix, known as uterus didelphys [4]. In most cases, a vaginal septum is also present. If this septum is obstructive on one side, this is almost invariably associated with ipsilateral renal or ureteric anomalies. To the best of our knowledge, only three cases of didelphic uterus and obstructed hemivagina with normal urinary tracts have been reported in the literature [5-7].

Although controversy exists concerning the embryogenesis of this malformation, the primum movens seems to be damage to the caudal portion of a mesonephric duct. Because the paramesonephric (Müllerian) ducts use the mesonephric (Wolffian) ducts to guide their descent toward the urogenital sinus, development of the Müllerian duct may be inhibited by a defective Wolffian duct, leading to anomalies in the fusion of both Müllerian ducts [8]. Capito et al. hypothesize that when an anomaly of the distal part of a Wolffian duct is present, a unilateral anomaly of incorporation of this duct within the bladder ensues, resulting in the anomalous incorporation of the attached ureteric bud within the bladder with subsequent ectopic (vaginal) ureteral ending. This situation would lead to a renal misdevelopment as the formation of the definitive kidney derives from cranial growth of the ureteral bud (the metanephric blastema is induced by the ureteric bud before it incorporates in the posterior wall of the bladder) $[9,10]$.

The pathogenesis of the obstructive hemivagina is also subject of discussion since the embryology of the upper vaginal portion remains elusive and controversial. If the upper vagina has a Müllerian (paramesonephric) origin, the failure of fusion of both the Müllerian ducts could lead to the malformative duct not reaching the urogenital sinus, thus impeding the resorption of the vaginal plate on the affected side. If the upper vagina has a Wolffian origin, the absence or distal agenesis of a mesonephric duct would result in lack of its opening into the urogenital sinus causing the blind hemivagina [10].

Typically, patients with an obstructing Müllerian anomaly present after menarche, with dysmenorrhea, severe pelvic or abdominal pain, or the presence of a pelvic or intraabdominal mass $[3,11,12]$. Other signs and symptoms vary depending on the individual patient's uterovaginal anatomy and may include fever, signs of intra-abdominal infection or purulent vaginal discharge (due to pyocolpos), urinary incontinence, and dyspareunia $[1,13,14]$.

Due to this heterogeneous clinical presentation and the low prevalence of the malformation, there is often a delay 
in diagnosis and subsequently a belated or suboptimal treatment. In addition, as only one uterine horn is obstructed, the diagnosis of outflow obstruction is less obvious compared to a patient presenting with amenorrhea and cyclic pain. Moreover, young patients with cyclic pelvic pain are often prescribed oral contraceptives and anti-inflammatory drugs, which might temporarily suppress the symptoms [1].

Zurawin et al. report a mean and median time to diagnosis of 37.8 weeks and 16 weeks, respectively, in their group of eight patients [1]. Another study by Capito et al. presents a series of 32 patients in which the median delay of diagnosis after menarche was 9 months (range 148 months). When there was a communication between the obstructed and the non-obstructed hemi tract, this period was even longer (median 20 months) [10].

However, for optimal outcome, early diagnosis is important. Delay in diagnosis can lead to infertility due to pelvic infection or adhesions. It has been demonstrated that endometriosis occurs more frequently in patients with didelphic uterus, probably due to retrograde menstruation [14]. A retrospective study of 41 cases of obstructed hemivagina found associated complications including hematosalpinx, pyosalpinx, pelvic adhesions, and endometriosis in $39 \%$ of cases [5].

When a didelphic uterus with obstructing hemivagina is suspected based on history and physical examination, detailed ultrasound examination can demonstrate the double uterus and the absence of the kidney on the side of the obstructed vagina $[1,14]$. It is important to note that, in cases of an ectopic ureter, the unilateral kidney is usually severely dysplastic, making it difficult to visualize the kidney on routine imaging studies [13].

MRI is the most sensitive non-invasive examination for evaluation of a Müllerian duct anomaly. Its accuracy in classifying uterine anomalies is reported to be $96 \%$ to $100 \%$, compared to $85 \%$ to $92 \%$ for transvaginal ultrasound and $6 \%$ to $55 \%$ for HSG [15]. It can define the fundal contour and visualize the uterus in different planes. Moreover, MRI gives excellent soft-tissue differentiation, therefore reliably distinguishing the endometrium from the myometrium [16]. Evaluation of Fallopian tubes and detection of intrauterine adhesions or mild endometriosis is difficult on MRI [15]. Therefore, hysteroscopy and laparoscopy should be considered for complete evaluation of the malformation and its possible complications. MRI then assists in the preoperative workup which allows the surgeon to develop the most appropriate surgical approach. If, during surgery, a didelphic uterus with obstructed hemivagina is confirmed, it may be treated instantly, as well as possible complications that might jeopardize future fertility.

Resection of the obstructive vaginal septum with marsupialization of the margins is the established treatment of didelphic uterus with blind hemivagina since drainage alone may lead to spontaneous closure and subsequent reformation of hemato- or pyocolpos [3, 8, 11]. This procedure allows evacuation of the sequestered material and preserves reproductive potential [8].

Classical surgical resection of a vaginal septum is performed after opening the septum by sequentially clamping, incising, and suturing the anterior and the posterior portions, using retractors for optimal visualization [13]. The edges must be sutured for hemostasis. This traditional surgical method is often difficult to perform in young patients because of the small size of the vulva and the vagina and the frequent high location of the septum. It requires careful attention to dissection and hemostasis to avoid injuries to bladder or rectum, bleeding, and scarring [12, 17].

Tsai et al. were the first to report a less invasive approach describing the vaginoscopic use of a resectoscope [18]. Using a needle-cutting electrode, they punched the vaginal septum and excised it progressively with a resectoscopic cutting electrode. The resectoscope permits exploration of the vaginal pathology of young patients with an intact hymen ring. The small diameter, the magnification, and the continuous fluid irrigation give ideal conditions for endoscopic evaluation of vaginal disorders in virgins [18]. The pressure caused by the irrigating solution offers a distended fibrous tissue to the surgeon, who can then easily cut the vaginal septum in the middle along a safe line, away from posterior or anterior structures - rectum or urethra. A Foley catheter placed in the bladder and a finger placed in the rectum will aid in delineating the correct anatomic relationships [1]. Once resected, the tissue retracts. As in hysteroscopic procedures for intrauterine septa, there is no need for suture [17].

Cicinelli et al. reported resectoscopic treatment with the use of a resectoscopic cutting loop under ultrasonographic guidance. Transabdominal ultrasonographic scanning allows a clear distinction between the vagina filled by the infusion fluid and the hematocolpos, both separated by the vaginal septum [12].

Patterson et al. described an alternative minimally invasive approach using a combination of laparoscopy and vaginoscopy, allowing for treatment in case of difficult differentiation of the septum from normal vaginal tissue. Importantly, the addition of laparoscopy allows for complete assessment of the uterine malformation as well as for associated complications affecting future fertility. It also allows for diagnosis of severe adnexal complications that may necessitate salpingectomy or hemi hysterectomy [11].

A unification operation for a didelphic uterus-Strassman procedure - is often not indicated. Not only is this procedure technically difficult but it is also unlikely to improve reproductive outcome and may result in cervical incompetence or cervical stenosis [2, 14]. 
Concerning treatment of the renal anomalies, it is important to bear in mind that a renal remnant with the ureter ending ectopic in the blind hemivagina, as in our case, could still secrete some urine. Therefore, performing an uretero-nephrectomy associated with the resection of the vaginal septum is recommended to prevent postoperative urinary incontinence [2, 10]. Some authors suggest an evaluation of the function of the ipsilateral and contralateral kidney in advance - e.g., by intravenous pyelogram or renal scintigraphy - to consider a ureteric reimplantation in case of functional renal parenchymal tissue [19]. However, in most cases described in the literature, no or minimal functional kidney is present, probably through a mechanism of dysplasia followed by non-functional hypoplasia [10]. For a blind ectopic ureter, no further procedure is required.

This case demonstrates the difficulty of establishing a correct diagnosis in adolescent women presenting with aspecific symptoms such as low grade fever and vulvodynia in a complex Müllerian anomaly. Prompt diagnosis and simple excision of the obstructed vaginal septum can completely relieve the symptoms and prevent further sequelae. A multidisciplinary approach by a team of gynecologists specialized in adolescent gynecology, operative hysteroscopy, and advanced laparoscopic surgery, and competent radiologists working in close cooperation with a dedicated family physician is the only way to ensure correct diagnosis and treatment.

Acknowledgements The authors wish to thank Ms. Amanda Sue McPhail for the language correction and the editing of the manuscript.

\section{References}

1. Zurawin RK, Dietrich JE, Heard MJ, Edwards CL (2004) Didelphic uterus and obstructed hemivagina with renal agenesis: case report and review of the literature. J Pediatr Adolesc Gynecol 17:137-141

2. Takashi S, Katsuya N, Hidehiro K, Masahide M, Toshimori S, Tomohiko K (1995) A case of unique communication between blind-ending ectopic ureter and ipsilateral hemi-hematocolpometra in uterus didelphys. J Urol 153:1208-1210

3. Stassart JP, Nagel TC, Prem KA, Phipps WR (1992) Uterus didelphys, obstructed hemivagina, and ipsilateral renal agenesis: the University of Minnesota experience. Fertil Steril 57(4):756761

4. Taylor E, Gomel V (2008) The uterus and fertility. Fertil Steril 89 (1):1-16

5. Haddad B, Barranger E, Paniel BJ (1999) Blind hemivagina: longterm follow-up and reproductive performance in 42 cases. Hum Reprod 14:1962-1964

6. Johnson J, Hillman BJ (1986) Uterine duplication, unilateral imperforate vagina, and normal kidneys. AJR Am J Roentgenol 147:1197-1198

7. Pinsonneault O, Goldstein DP (1985) Obstructing malformations of the uterus and vagina. Fertil Steril 44:241-247

8. Candiani GB, Fedele L, Candiani M (1997) Double uterus, blind hemivagina, and ipsilateral renal agenesis: 36 cases and long-term follow-up. Obstet Gynecol 90:26-32

9. Vercellini P, Daguati R, Somigliana E, Vigano P, Lanzani A, Fedele L (2007) Asymmetric lateral distribution of obstructed hemivagina and renal agenesis in women with uterus didelphys: institutional case series and a systematic literature review. Fertil Steril 87(4):719-724

10. Capito C, Echaieb A, Lortat-Jacob S, Thibaud E, Sarnacki S, Nihoul-Fékété C (2008) Pitfalls in the diagnosis and management of obstructive uterovaginal duplication: a series of 32 cases. Pediatrics 122(4):e891-e897

11. Patterson D, Mueller C, Strubel N, Rivera R, Ginsburg HB, Nadler EP (2006) Laparoscopic neo-os creation in an adolescent with uterus didelphys and obstructed hemivagina. J Pediatr Surg 41:E19-E22

12. Cicinelli E, Romano F, Didonna T, Schonauer LM, Galantino P, Di Naro El (1999) Resectoscopic treatment of uterus didelphys with unilateral imperforate vagina complicated by hematocolpos and hematometra: case report. Fertil Steril 72:553-555

13. Hansen KA, DeWitt J (2005) Premenarchal, recurrent vaginal discharge associated with an incomplete obstructing longitudinal vaginal septum. J Pediatr Adolesc Gynecol 18:423-426

14. Frei KA, Bonel HM, Häberlin FC (1999) Uterus didelphys, obstructed hemivagina and ipsilateral renal agenesis with excessive chronic vaginal discharge. Acta Obstet Gynecol Scand 78:460-461

15. Pui MH (2004) Imaging diagnosis of congenital uterine malformation. Comput Med Imaging Graph 28:425-433

16. Takagi H, Matsunami K, Noda K, Furui T, Imai A (2003) Magnetic resonance imaging in the evaluating of double uterus and associated urinary tract anomalies: a report of five cases. J Obstet Gynaecol 23(5):525-527

17. Montevecchi L, Valle RF (2004) Resectoscopic treatment of complete longitudinal vaginal septum. Int J Gynaecol Obstet 84 (1):65-70

18. Tsai EM, Chiang PH, Hsu SC et al (1998) Hysteroscopic resection of vaginal septum in an adolescent virgin with obstructed hemivagina. Hum Reprod 13:1500-1501

19. Dwyer PL, Rosamilia A (2006) Congential urogenital anomalies that are associated with the persistence of Gartner's duct: a review. Am J Obstet Gynecol 195:354-359 